Check for updates

Cite this: RSC Adv., 2018, 8, 1047

Received 24th September 2017 Accepted 13th December 2017

DOI: 10.1039/c7ra10574f

rsc.li/rsc-advances

\section{Thermal and mechanical reinforcement of a novel paraffin-based hydroxyl-terminated polybutadiene (HTPB) binder containing a three-dimension (3D) diurea-paraffin wax (DU-PW) for prevention of PW leakage $\dagger$}

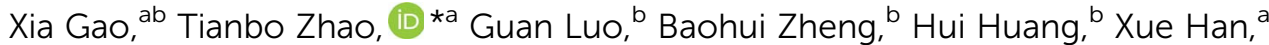 \\ Rui $\mathrm{Ma}^{\mathrm{a}}$ and Yuqiao $\mathrm{Chai}^{\mathrm{a}}$
}

Leakage of paraffin wax (PW) is a major concern in the development of polymer bonded explosive (PBX) systems because it relates to the amount of PW that can be used as a desensitizer or a fuel, which, in turn, affects the mechanical performance and tolerance of PBX in high-temperature environments. Hydroxyl-terminated polybutadiene (HTPB) binders significantly contribute desirable polymer features to PBX. Thus, a three-dimension (3D) high-temperature non-flowing diurea-paraffin wax (DU-PW) composite was synthesized and creatively employed to a HTPB binder. DU-PW/PW/HTPB composites with different contents of the 3D DU-PW phase change material (PCM) were prepared through a cast molding process. The differential scanning calorimetry (DSC) results demonstrate that these composites can show high phase-change enthalpies and good thermal reliability. As observed from the scanning electron microscope (SEM) photographs, adding DU-PW can clearly reduce the number of holes caused by the leaked PW on the fracture surface of DU-PW/HTPB. Moreover, the addition of DU-PW can remarkably reduce the leakage of $\mathrm{PW}$ and improve the thermal stability as well as mechanical properties of the PW-based HTPB. These observations present the potential of utilizing form-stable PCM (FSPCM) to solve the problem of PW leakage in PBX systems.

\section{Introduction}

Polymer bonded explosives (PBX) are promoted for insensitive munitions, consisting of explosive crystals, polymer binders, desensitizers, plasticizers, thermal stabilizers and some other functional additives. ${ }^{1-4}$ Polymer binders play an important role as structural supporting materials and endow PBX with desirable properties of polymers, such as cast-ability and machineability. ${ }^{5,6}$ In the past few decades, hydroxyl-terminated polybutadiene (HTPB) has been widely used as a binder in military engineering due to its superior mechanical and ballistic properties. $^{7,8}$ In addition, HTPB-derived polyurethane can provide a matrix of networks to impart dimensional stability and structural integrity to $\mathrm{PBX} .{ }^{9}$ With the increasing attention on the trade-off between high performance and low sensitivity of

${ }^{a}$ Key Laboratory of Cluster Science of Ministry of Education, Beijing Institute of Technology, Beijing 100081, PR China. E-mail: bit_bipt@126.com; Tel: +86-10-81381389

${ }^{b}$ Institute of Chemical Materials, China Academy of Engineering Physics (CAEP), Mianyang 621900, PR China

$\dagger$ Electronic supplementary information (ESI) available. See DOI: $10.1039 / \mathrm{c} 7 \mathrm{ra} 10574 \mathrm{f}$ energetic materials, ${ }^{\mathbf{1 0 , 1 1}}$ paraffin wax (PW) serves as a promising desensitizer and solid fuel ${ }^{12}$ due to its low cost, controllable phase-transition temperature, chemical stability, environmentfriendliness, high heat of combustion and burning regression rate. ${ }^{12-15}$ Jin et $a l .{ }^{16}$ studied the coating and desensitization effects of $\mathrm{PW} /$ stearic acid (SA) composite on 2,4,6,8,10,12hexanitro-2,4,6,8,10,12-hexaazaisowurtzitane (HNIW). Kawachi et $a .^{13}$ investigated the thermal and mechanical tests of a combustible grain (PW/HTPB) casted with maximum loading; the results indicated that this material could meet the criteria of performance and safety required for this type of a combustible grain. However, poor mechanical properties, diffusion and leakage of melted PW will always be an issue if the PW is directly suspended in HTPB using traditional methods. ${ }^{17}$ Therefore, prevention of $\mathrm{PW}$ leakage is the main topic of most of these investigations.

PW is usually categorized as an organic solid-liquid phase change material (PCM). In recent years, microencapsulated PCM (MEPCM) and form-stable PCM (FSPCM) have proved to be effective techniques to prevent the leakage in PCM. Compared with FSPCM, MEPCM with shell materials requires complicated synthesis procedures, high volume change, large super-cooling and high cost, which hinder its application. ${ }^{18-20}$ FSPCM is 
prepared by absorbing PCM with various polymers and inorganic porous materials as supporting materials, including polyethylene glycol (PEG), ${ }^{21}$ high density polyethylene (HDPE), ${ }^{22}$ epoxy, ${ }^{23}$ polyurethane (PU) and PU foam, ${ }^{24-26}$ porous metal fiber sintered felt (PMFSF), ${ }^{27}$ multi-walled carbon nanotubes (MWCNTs), ${ }^{28}$ graphene, ${ }^{29}$ nanoplatelet graphite, ${ }^{30}$ activated carbon (AC), ${ }^{31}$ expanded graphite (EG) ${ }^{32}$ and fibers. ${ }^{33,34}$ Samui et $a l .{ }^{35}$ developed a microwave technology to prepare a shapestabilized PEG-cellulose acetate blend with high PEG loading. Karaipekli et $a .^{36}$ prepared an expanded perlite (EP)/paraffin composite PCM with highly enhanced thermal conductivity using carbon nanotubes. Although these supporting materials can effectively shape-stabilize PW during its phase transition, the temperature range required to keep $\mathrm{PW}$ form-stable is too narrow for its application in higher-temperature environments. Lubricating grease, a type of high-temperature material such as cream, is generally prepared by thickening base oil with polyurea. ${ }^{37}$ Thus, our team introduced diurea (DU) as a new supporting material to absorb PW owing to its simple preparation process, high stability, amphipathy and surface activity.

In order to find a solution to PW leakage in HTPB binders and inspired by the recent progress in FSPCM, a hightemperature DU-PW composite is creatively employed to the HTPB matrix. In this study, it is meaningful to investigate the effect of different loadings of the DU-PW composite on the leakage, thermal and mechanical properties of the DU-PW/PW/ HTPB composite because these HTPB binder composites with stable shapes can be employed for the practical application of PBX systems.

\section{Experiment}

\subsection{Materials}

Paraffin wax $\left(T_{\mathrm{m}}=50-52{ }^{\circ} \mathrm{C}\right)$ was purchased from Shanghai Huashen Rehabilitation Material Co., Ltd., China. Octadecylamine (CP) was supplied by the Research Institute of Tianjin Guangfu Fine Chemical Industry Co., Ltd., China. Paratoluidine (AR) was commercially obtained from Shanghai Macklin Chemical Co., Ltd., China. Bis(4-isocyanatophenyl)methane (MDI, 98\%) was supplied by J\&K Chemical Co., Ltd., China. Petroleum ether (AR) was purchased from Chongqing Chuandong Chemical Co., Ltd., China.

Hydroxylterminated polybutadiene (HTPB) $(\mathrm{OH}$ value $=$ $0.76 \mathrm{mmol} \mathrm{g}^{-1}$ ) was purchased from Liming Research \& Design Institute of Chemical Industry Co., Ltd., China. Toluene diisocyanate (TDI) (AR) was supplied by Xiya Chemical Industry Co., Ltd., China. Dinoctylsebacate (DOS) (AR) was purchased from Jiangyin Bolong Chemical Group Co., Ltd.

\subsection{Preparation of DU-PW composite}

The preparation process of the DU-PW composite can be described as follows. Octadecylamine and paratoluidine were mixed with partial PW at $79{ }^{\circ} \mathrm{C}$. Simultaneously, MDI was exhaustively blended with PW in a container at $79{ }^{\circ} \mathrm{C}$. Then, the MDI blend was poured into an organic amine mixture quickly and agitated at $99^{\circ} \mathrm{C}$ for $0.5 \mathrm{~h}$. Subsequently, the temperature of the oil bath increased to $190{ }^{\circ} \mathrm{C}$ at the rate of $2{ }^{\circ} \mathrm{C} \mathrm{min}{ }^{-1}$. The mixture was continuously agitated for $1 \mathrm{~h}$ and naturally cooled to room temperature. Finally, the DU-PW composite $(\mathrm{DU} / \mathrm{PW}=$ 2/8) was prepared. The photographs of pristine PW and the prepared DU-PW composite are shown in Fig. S1. $\dagger$ To obtain the DU particles without PW, DU-PW was extracted with petroleum ether for $48 \mathrm{~h}$, repeatedly centrifuged, and dried at $25{ }^{\circ} \mathrm{C}$ for $24 \mathrm{~h}$.

\subsection{Preparation of DU-PW/PW/HTPB composites}

In the preparation process of the DU-PW/PW/HTPB composite, the DU-PW composite was intended to replace $20 \mathrm{wt} \%, 40 \mathrm{wt} \%$, $60 \mathrm{wt} \%$, and $100 \mathrm{wt} \%$ of PW in the HTPB matrix. The HTPB (324.17 g) was blended with DOS (154.95 g), TDI (20.88 g), PW and DU-PW at $60{ }^{\circ} \mathrm{C}$ for $30 \mathrm{~min}$. The defined amount of PW and DU-PW is presented in Table S1. $\dagger$ Then, the mixture was placed in a vacuum oven at $60{ }^{\circ} \mathrm{C}$ for $30 \mathrm{~min}$ to remove air bubbles. Subsequently, the mixture was poured into aluminum molds with different specimen dimensions. Finally, these molds were cured in an oven at $65{ }^{\circ} \mathrm{C}$ for 3 days and cooled to room temperature. The photographs of pristine HTPB, the PW/HTPB composite and the DU-PW/HTPB composite are shown in Fig. S2. $\dagger$

\subsection{Characterizations}

The chemical composition of the DU-PW and DU-PW/PW/ HTPB composites was investigated by Fourier transformed infrared spectroscopy (FT-IR, Nicolet 6700), within a wave number range of $350-4000 \mathrm{~cm}^{-1}$.

The microstructure of the DU particles and the DU-PW/PW/ HTPB composite samples was studied using a scanning electron microscope (SEM, ZEISS ULTRA 55). The DU-PW/PW/HTPB samples were prepared by cryofracturing in liquid nitrogen. The cross sections of the composites and the DU particles were sputter-coated with gold before analysis.

The thermal stability of the DU-PW and DU-PW/PW/HTPB composites was studied using a thermal gravimetric analyzer (TGA, TG-DTA $6200 \mathrm{LAB}$ SYS) with a heating rate of $10^{\circ} \mathrm{C} \mathrm{min}^{-1}$ from $25{ }^{\circ} \mathrm{C}$ to $600{ }^{\circ} \mathrm{C}$ in a stream of nitrogen.

The phase change properties of DU-PW and DU-PW/PW/ HTPB composites were studied using a differential scanning calorimeter (DSC, DSC Q2000 V24.11 Build 124) with a temperature range from -10 to $75{ }^{\circ} \mathrm{C}$ at a heating rate of $10{ }^{\circ} \mathrm{C} \mathrm{min}{ }^{-1}$, in a nitrogen stream. An accelerated 500 thermal cycling test $\left(25-75{ }^{\circ} \mathrm{C}\right)$ was conducted in a programmable high-low temperature test chamber.

The compression test of the DU-PW/PW/HTPB samples with dimensions of $\Phi 20 \mathrm{~mm} \times 20 \mathrm{~mm}$ was characterized according to ISO 7743:2007 using an MTS Criterion Model 45 electromechanical universal testing machine. The crosshead speed was set to $5 \mathrm{~mm} \mathrm{~min}^{-1}$. For each sample, the average value of five data was taken.

The tensile test of the DU-PW/PW/HTPB samples was measured on a MTS Criterion Model 45 electromechanical universal testing machine with a crosshead speed of 5 $\mathrm{mm} \min ^{-1}$ according to ISO 37:2005. 
Leaking test on the samples was performed in a cylinder placed in an oven for 30 days at $75{ }^{\circ} \mathrm{C}$. The samples with dimension of $\Phi 24 \mathrm{~mm} \times 125.75 \mathrm{~mm}$ were packed using a filter paper. To get a quantitative value of the PW leakage, the initial and final weights of the samples and the filter paper were recorded and calculated.

\section{Results and discussions}

\subsection{Scheme and structure of DU-PW/HTPB composite}

The schematic illustration of the synthesis process of the DUPW/HTPB binder composite is shown in Fig. 1. As presented in Fig. 1, the organic amines (octadecylamine and paratoluidine) react with MDI and produce the DU molecules. The DU molecules self-assemble into a three-dimensional (3D) supramolecular structure by intermolecular interaction. Then, the DU-PW composite was synthesized by absorbing PW into the 3D supramolecular structure of DU. Subsequently, the DU-PW composite was blended with the HTPB matrix to prepare the DU-PW/HTPB composite. In this composite, the 3D supramolecular structure of DU is formed like a cage to lock the PW molecules, which is intended to stabilize the PW. Thus, the process of introducing DU-PW into the HTPB matrix can prevent the leakage of PW.

\subsection{Chemical composition of DU-PW and DU-PW/HTPB composites}

The FTIR spectra of PW, DU, DU-PW, HTPB and DU-PW/HTPB composites are presented in Fig. 2. As observed in Fig. 2, in the

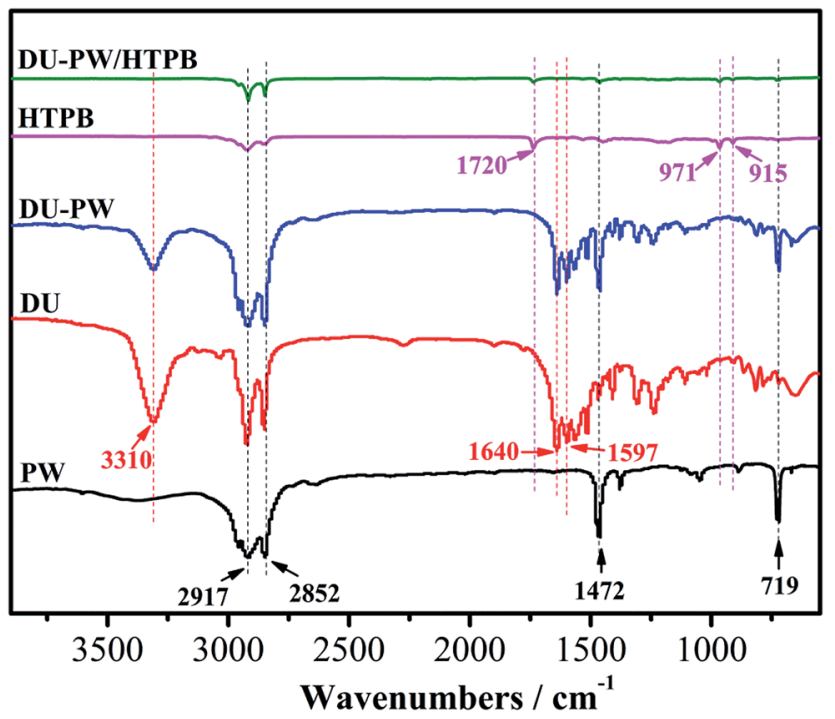

Fig. 2 FTIR spectra of pristine PW, DU, DU-PW composite, pristine HTPB and DU-PW/HTPB composites.

spectrum of PW, the peaks at 2917 and $2852 \mathrm{~cm}^{-1}$ were attributed to the aliphatic $\mathrm{C}-\mathrm{H}$ stretching vibration of the $-\mathrm{CH}_{3}$ and $-\mathrm{CH}_{2}$ groups, ${ }^{38}$ respectively. The peaks at 1462 and $719 \mathrm{~cm}^{-1}$ were ascribed to the bending and in-plane rocking vibration of the $\mathrm{CH}_{2}$ groups. ${ }^{39}$ For the DU sample, the absorption peak at $3310 \mathrm{~cm}^{-1}$ was assigned to the stretching vibration of the hydrogen bonded $\mathrm{N}-\mathrm{H}$ group. The peak located at $1597 \mathrm{~cm}^{-1}$ was assigned to the bending vibration absorption of the $\mathrm{N}-\mathrm{H}$

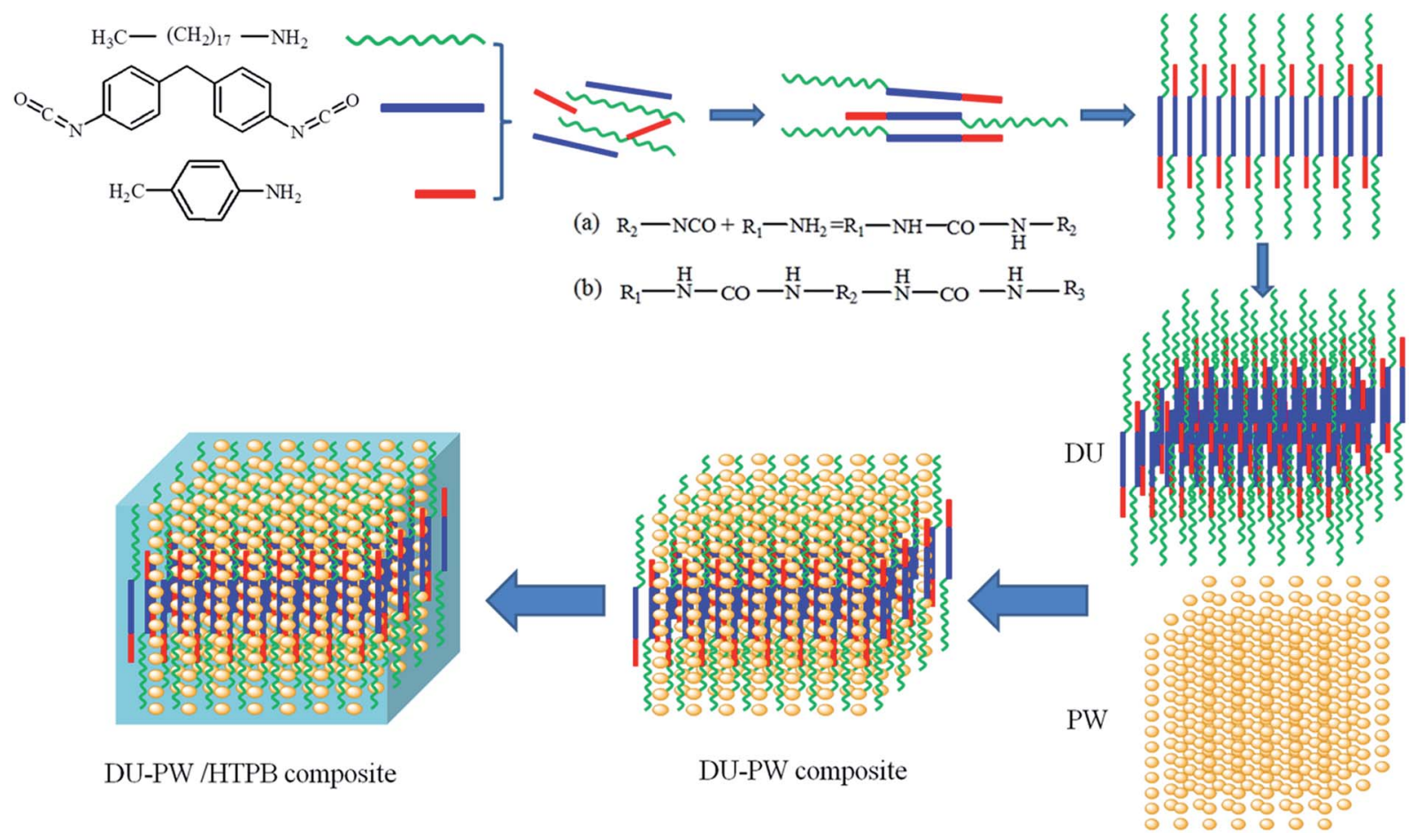

Fig. 1 Schematic illustration of the synthesis process of DU-PW/HTPB binder composite: $R_{1}, R_{3}$ are alkyl, cycloalkyl or aryl; $R_{2}$ is arylene, alkylene or cycloalkylene. 

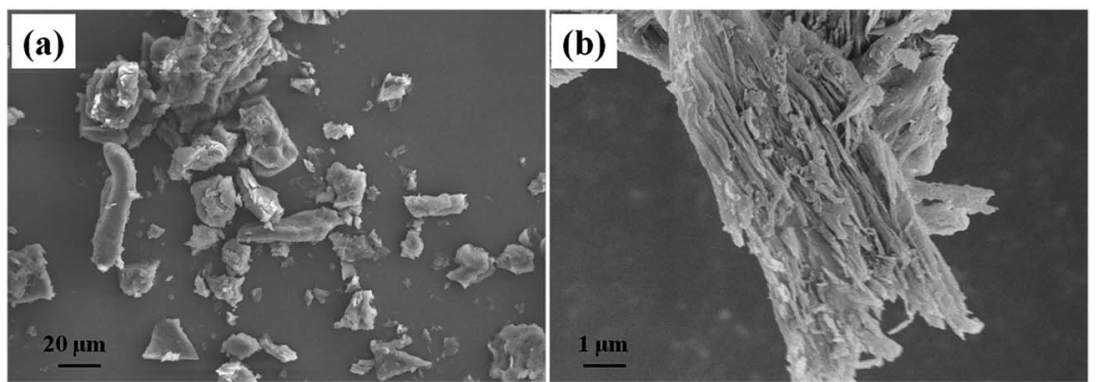

Fig. 3 SEM micrographs of DU particles.

group. The characteristic absorption peak of $-\mathrm{C}=\mathrm{O}$ group was found at $1640 \mathrm{~cm}^{-1}$, due to the hydrogen bond between the DU molecules. In the spectrum of the DU-PW composite, the characteristic peaks of PW and DU can be observed. This indicated that the DU-PW composite has been successfully synthesized without any chemical reaction. For HTPB, the peak at $1720 \mathrm{~cm}^{-1}$ belonged to the urethane groups and the absorption peaks at 971 and $915 \mathrm{~cm}^{-1}$ were corresponding to the bending vibrations of the $\mathrm{C}=\mathrm{C}$ band. ${ }^{6}$ Compared with pristine HTPB, the spectrum of the DU-PW/HTPB composite was almost consistent, suggesting that no chemical interaction occurred between the two components. The results indicated that the DU-PW/HTPB composite had been prepared through physical blending.

\subsection{Morphology of DU and DU-PW/PW/HTPB composites}

The morphology of the DU particles without PW is shown in Fig. 3. In Fig. 3a, the DU particles exhibited an irregular shape with a diameter range of about $10-100 \mu \mathrm{m}$. As observed from Fig. $3 \mathrm{~b}$, there are numerous $3 \mathrm{D}$ networks of fibrous and striplike structures existing in DU particles, which would trap the movement of melted PW and support PW to FSPCM. Therefore, the DU particles with a gel network structure could serve as a new supporting material. ${ }^{40}$ The microstructures of the fracture surfaces of the HTPB and DU-PW/PW/HTPB composites are shown in Fig. 4. As shown in Fig. 4a, there are some rupture cracks on the smooth fracture surface of pristine HTPB due to the brittle failure of HTPB in liquid nitrogen. For the PW/HTPB composite, numerous dark holes and spherical particles are distributed on the fracture surface. These holes may be left by the exuded PW particles, which were displayed as spherical particles. With the addition of the DU-PW composite, fewer holes and PW particles could be observed on the fracture surface of the DU-PW/PW/HTPB composite since the selfassembled 3D DU could make a thickened entanglement with the pure PW and HTPB. ${ }^{40}$ Therefore, few holes and PW particles exist on the fracture surface of the DU-PW/HTPB composite. This indicated that the addition of the DU-PW composite can effectively prevent the separation of PW from the HTPB matrix.

\subsection{Phase change properties of DU-PW/PW/HTPB composites}

The phase change properties of PW, DU-PW, pristine HTPB, $\mathrm{PW} / \mathrm{HTPB}$ and DU-PW/PW/HTPB composites are presented in
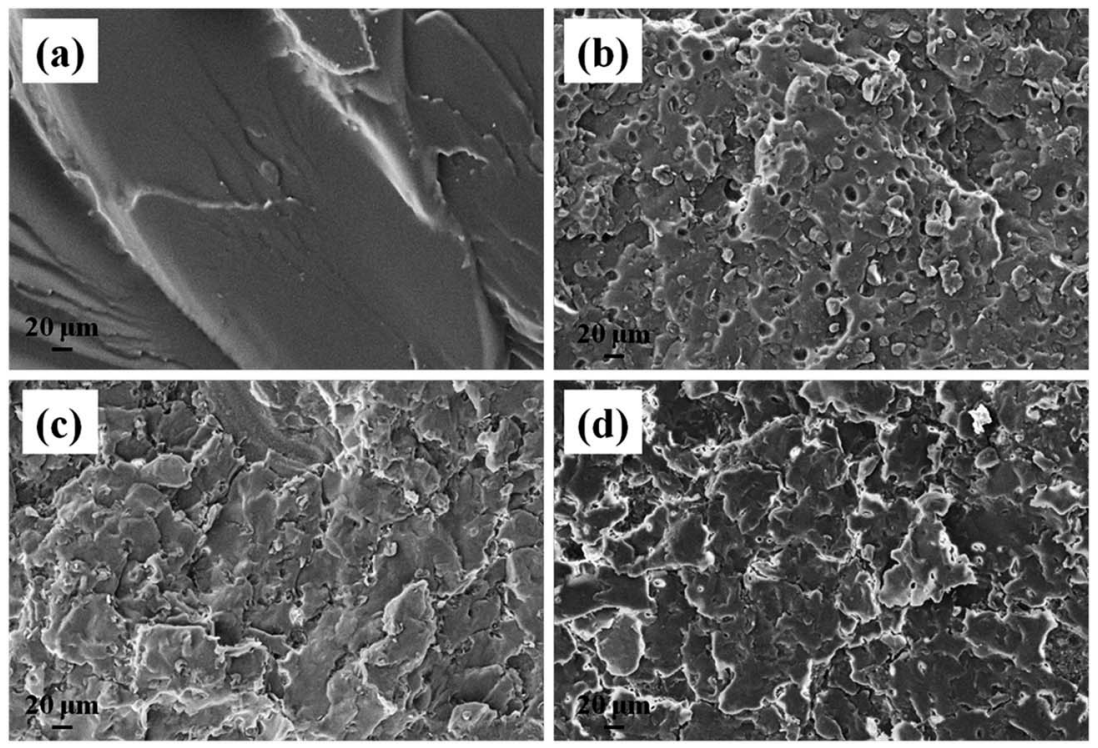

Fig. 4 SEM micrographs of (a) pristine HTPB, (b) PW/HTPB, (c) 60 wt\% DU-PW/PW/HTPB and (d) DU-PW/HTPB composites. 

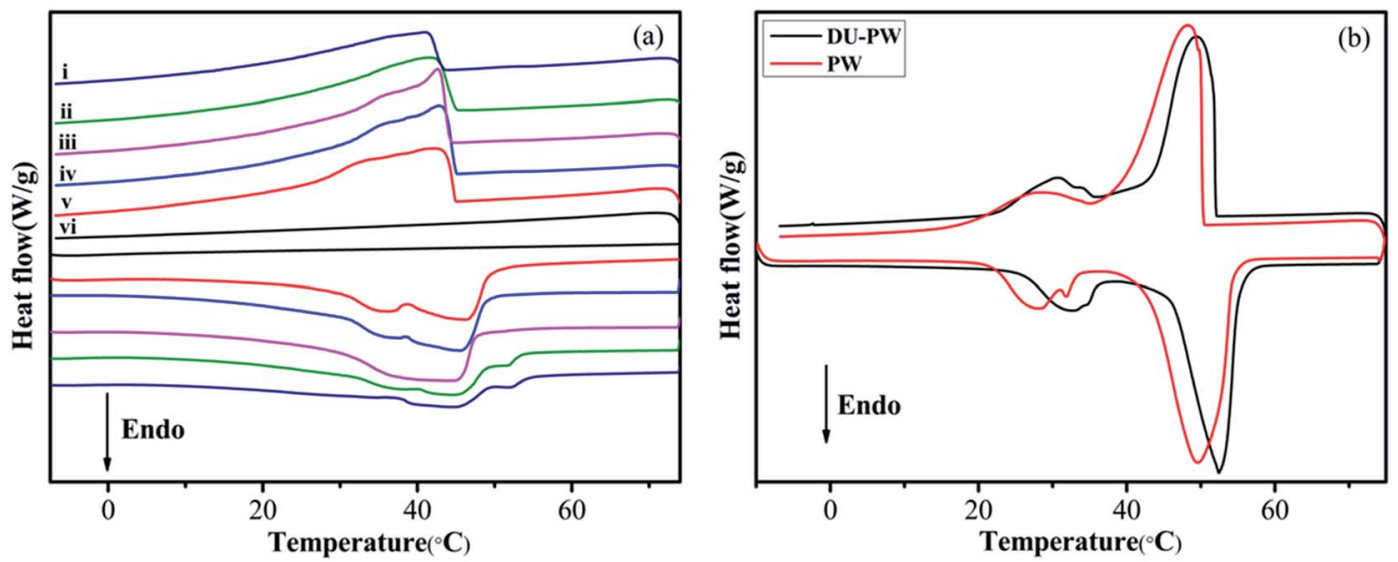

Fig. 5 DSC curves of (a-i) DU-PW/HTPB, (a-ii) 60 wt\% DU-PW/PW/HTPB, (a-iii) 40 wt\% DU-PW/PW/HTPB, (a-iv) 20 wt\% DU-PW/PW/HTPB, (a-v) PW/HTPB, and (a-vi) pristine HTPB, (b) PW and DU-PW composites.

Fig. 6 and summarized in Table S2. $\dagger$ As displayed in Fig. 5a, there is no phase change peak on the DSC curve of the HTPB matrix, indicating that no phase change occurred in HTPB within this temperature range. For the DU-PW/PW/HTPB composites, phase change occurred during the melting and freezing processes, caused by the addition of PW and DU-PW. In Fig. $5 \mathrm{~b}$, the temperatures at the endothermic peak $\left(T_{\mathrm{m}}\right)$ and exothermic peak $\left(T_{\mathrm{c}}\right)$ of DU-PW were improved compared with PW due to the shape-stabilizing effect of DU on PW. This would delay the phase change process of PW. As listed in Table S2, $\dagger$ the difference $\left(\Delta T_{\mathrm{s}}\right)$ between the $T_{\mathrm{m}}$ and $T_{\mathrm{c}}$ of DU-PW/PW/HTPB composites was lower than that of PW/HTPB, indicating that DU-PW can limit the super-cooling phenomena of PW-based HTPB. As the content of DU-PW increased, the calculated phase change enthalpies $\left(\Delta H_{2}\right)$ of DU-PW/PW/HTPB composites decreased because the total content of pure PW in HTPB decreased. In addition, the $\Delta H_{1}$ of most DU-PW/PW/HTPB composites was lower than $\Delta H_{2}$; owing to that the curing process $\left(65^{\circ} \mathrm{C}, 3\right.$ days) would lead to the diffusion of PW in the composites. However, the $\Delta H_{1}$ of $20 \mathrm{wt} \%$ and $40 \mathrm{wt} \% \mathrm{DU}-\mathrm{PW} /$ $\mathrm{PW} / \mathrm{HTPB}$ composites was slightly higher than that of $\mathrm{PW} /$ HTPB. It helps to illustrate that the DU can hold the PW firmly in the HTPB matrix. Moreover, the difference (error\%) between $\Delta H_{1}$ and $\Delta H_{2}$ of the DU-PW/PW/HTPB composites was much lower than that of PW/HTPB, implying that less diffusion of PW took place in DU-PW/PW/HTPB than that in PW/HTPB. This indicates that the DU-PW/PW/HTPB composites exhibit high heat capacity and higher form-stability than PW/HTPB due to the shape-stabilizing function of DU-PW.

\subsection{Thermal stability of DU-PW/PW/HTPB composites}

TGA was used to investigate the thermal stability of the DU-PW/ PW/HTPB composites. The relevant results are presented in Fig. 6 and Table 1. As shown in Fig. 6a, there is one weight loss stage in the TGA curves of PW and DU-PW due to the evaporation and decomposition of PW and DU. Compared with PW, the $T_{1,0}, T_{1, \mathrm{e}}$ and $T_{1, \mathrm{~m}}$ of the DU-PW composite dramatically increased by $3.78,75.63$ and $51.31{ }^{\circ} \mathrm{C}$, respectively, due to that the DU molecules could absorb and shape-stabilize PW to delay the evaporation of PW. ${ }^{41}$ The total weight loss of DU-PW was lower than that of PW due to the additional DU molecules. Thus, the total weight loss of DU-PW/HTPB was slightly lower than that of PW/HTPB. In the TGA curve of pristine HTPB, there are two decomposition stages, corresponding to the
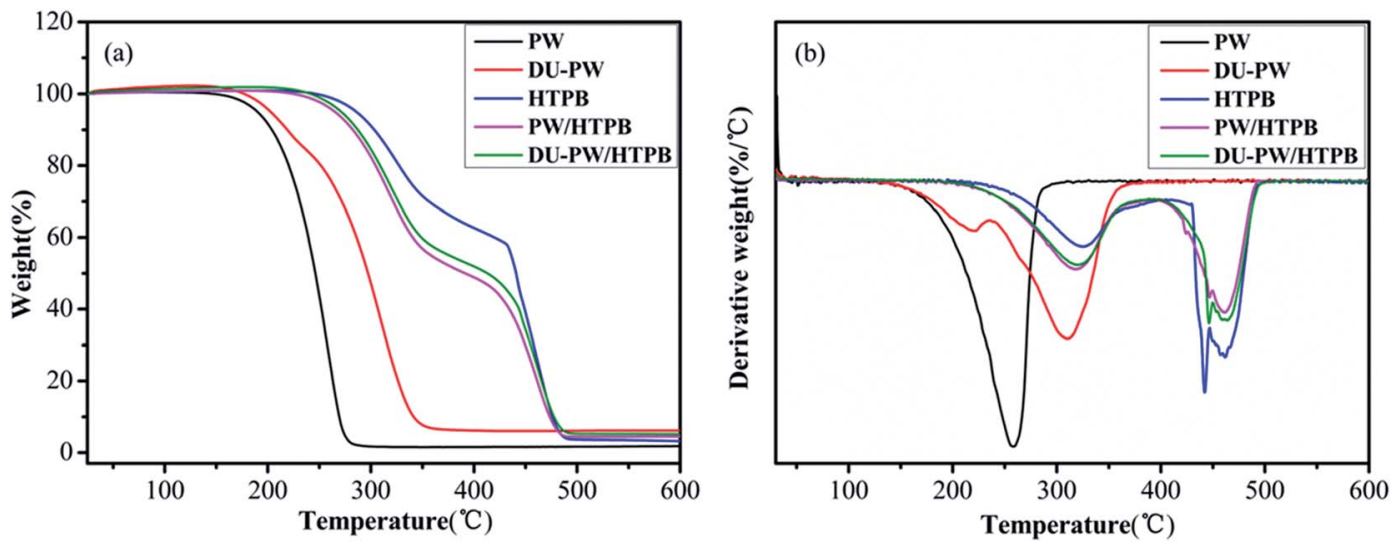

Fig. 6 (a) TGA and (b) DTG curves of PW, DU-PW, pristine HTPB, PW/HTPB and DU-PW/HTPB composites. 
Table 1 Thermal stability of PW, DU-PW, pristine HTPB, PW/HTPB and DU-PW/HTPB composites ${ }^{a}$

\begin{tabular}{|c|c|c|c|c|c|c|c|c|c|}
\hline Samples & \multicolumn{4}{|l|}{ Step 1} & \multicolumn{4}{|l|}{ Step 2} & $\begin{array}{l}\text { Total weight } \\
\text { loss (\%) }\end{array}$ \\
\hline PW & 145.39 & 298.81 & 259.19 & 98.12 & - & - & - & - & 98.12 \\
\hline DU-PW & 149.17 & 374.44 & 310.50 & 93.21 & - & - & - & - & 93.21 \\
\hline НТРВ & 212.83 & 402.83 & 324.83 & 28.02 & 418.83 & 511.25 & 461.17 & 68.33 & 96.35 \\
\hline
\end{tabular}

${ }^{a} T_{1,0}$ and $T_{1, \mathrm{e}}$ were determined from the cross-point of two tangent lines at related bending locations during the first rapid decomposition. $T_{1, \mathrm{~m}}$ was determined as the temperature at the peak on the derivative curve of the first decomposition. Similar methods were adopted to obtain $T_{2,0}, T_{2, \mathrm{e}}$ and $T_{2, \mathrm{~m}}$.

depolymerization and decomposition of HTPB. ${ }^{42}$ For the PW/ HTPB composite sample, there are also two weight loss stages in the TGA curve. The first weight loss stage is attributed to the evaporation of PW and partial decomposition of HTPB. The second stage is ascribed to the decomposition of HTPB. Moreover, the introduction of PW in the PW/HTPB composite resulted in a decrease in the total weight loss compared with the HTPB matrix. It is noteworthy that the $T_{1,0}, T_{1, \mathrm{e}}$ and $T_{1, \mathrm{~m}}$ of the DU-PW/ HTPB composite are higher than those of PW/HTPB as expected. Therefore, it was demonstrated that the introduction of DU-PW can improve the thermal stability of the PW/HTPB composite.

\subsection{Thermal reliability of DU-PW/PW/HTPB composites}

A 500-thermal-cycle test was carried out to investigate the thermal reliability of the DU-PW/HTPB composites. The DSC curves of PW/HTPB and DU-PW/HTPB composites before and after thermal cycling are shown in Fig. 7. As observed in Fig. 7, the temperatures at the melting and solidifying peaks of the $\mathrm{PW} /$ HTPB and DU-PW/HTPB samples are almost consistent before and after repetitive thermal cycles. The phase change enthalpies of the PW/HTPB and DU-PW/HTPB composites slightly decreased after 500-thermal cycles due to the diffusion of PW during the long-time thermal cycling process. This result illustrated that the DU-PW/HTPB composite exhibited good thermal reliability.

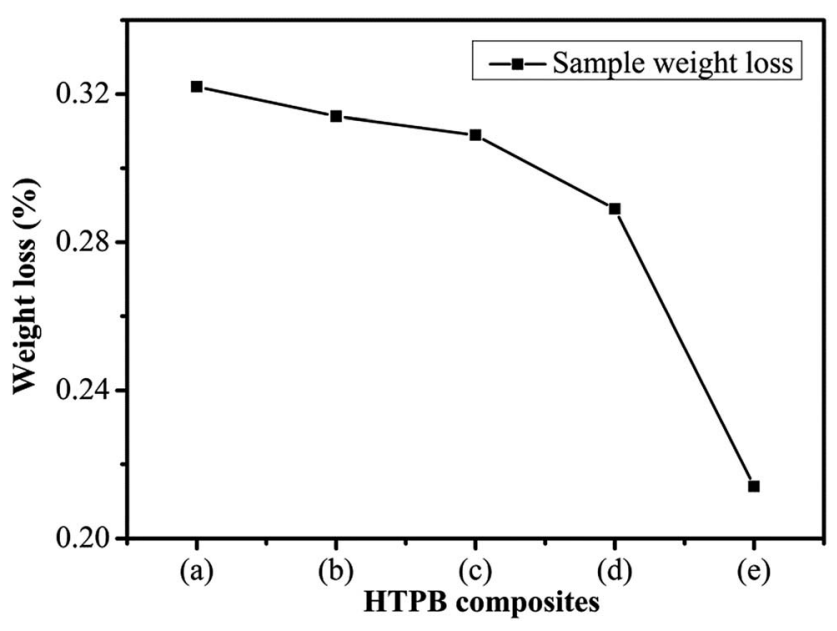

Fig. 8 Sample weight loss of (a) PW/HTPB, (b) 20 wt\% DU-PW/PW/ HTPB, (c) 40 wt\% DU-PW/PW/HTPB, (d) 60 wt\% DU-PW/PW/HTPB, (e) DU-PW/HTPB composite.

\subsection{Leakage of DU-PW/PW/HTPB composites}

The weight loss of the PW/HTPB, DU-PW/PW/HTPB and DU-PW/HTPB composites is shown in Fig. 8. As observed from the weight loss curve in Fig. 8, with increasing contents of
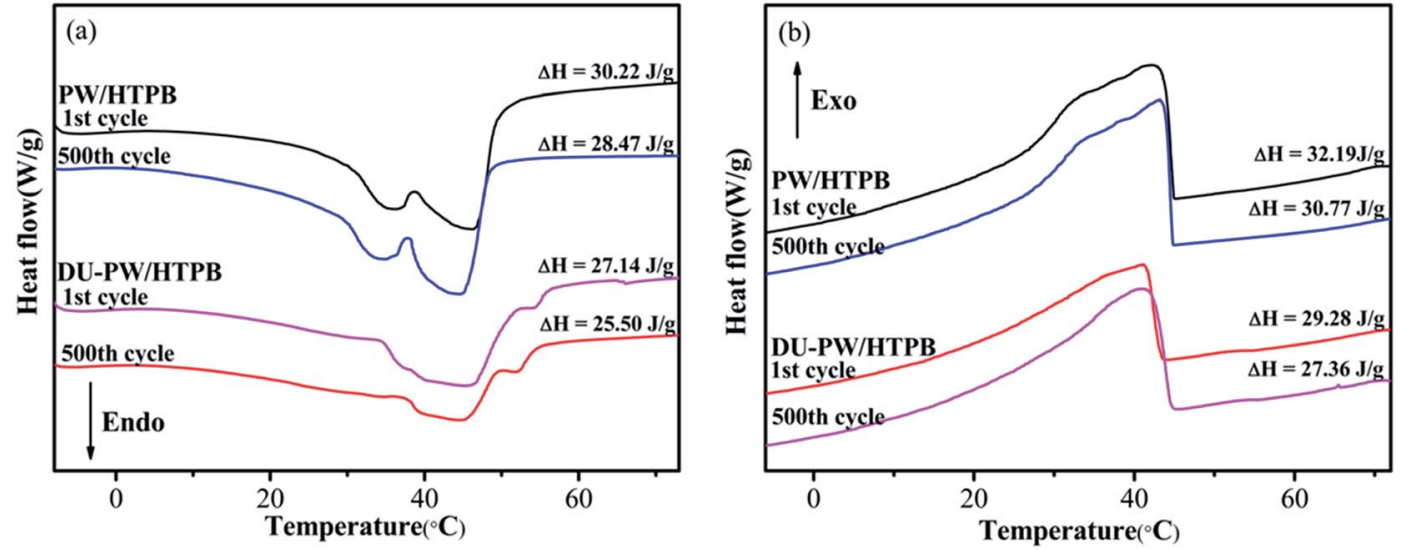

Fig. 7 (a) Melting and (b) solidifying DSC curves of PW/HTPB and DU-PW/HTPB composites before and after 500-thermal-cycle test. 
Table 2 Mechanical properties of pristine HTPB, PW/HTPB, DU-PW/PW/HTPB and DU-PW/HTPB composites

\begin{tabular}{|c|c|c|c|c|c|c|}
\hline Samples & $\begin{array}{l}\text { Compressive } \\
\text { modulus }^{a}(\mathrm{MPa})\end{array}$ & $\begin{array}{l}\text { Compressive } \\
\text { strength }^{a}(\mathrm{MPa})\end{array}$ & $\begin{array}{l}\text { Compressive } \\
\operatorname{strain}^{b}(\%)\end{array}$ & $\begin{array}{l}\text { Tensile } \\
\text { modulus }^{c}(\mathrm{MPa})\end{array}$ & $\begin{array}{l}\text { Tensile } \\
\text { strength }^{d}(\mathrm{MPa})\end{array}$ & $\begin{array}{l}\text { Elongation } \\
\text { at break (\%) }\end{array}$ \\
\hline НТРВ & $0.0123 \pm 0.0003$ & $0.056 \pm 0.004$ & $87.30 \pm 5.89$ & $0.014 \pm 0.003$ & $0.264 \pm 0.024$ & $343.88 \pm 26.047$ \\
\hline PW/HTPB & $0.0118 \pm 0.0007$ & $0.061 \pm 0.004$ & $84.62 \pm 3.61$ & $0.015 \pm 0.005$ & $0.252 \pm 0.041$ & $294.28 \pm 35.79$ \\
\hline 20 wt\% DU-PW/PW/HTPB & $0.0132 \pm 0.0006$ & $0.150 \pm 0.004$ & $78.33 \pm 0.87$ & $0.018 \pm 0.001$ & $0.249 \pm 0.021$ & $378.19 \pm 7.52$ \\
\hline $40 \mathrm{wt} \%$ DU-PW/PW/HTPB & $0.0181 \pm 0.003$ & $0.155 \pm 0.005$ & $77.76 \pm 0.34$ & $0.019 \pm 0.003$ & $0.250 \pm 0.034$ & $407.94 \pm 10.27$ \\
\hline $60 \mathrm{wt} \%$ DU-PW/PW/HTPB & $0.0230 \pm 0.0006$ & $0.156 \pm 0.007$ & $78.43 \pm 0.23$ & $0.019 \pm 0.002$ & $0.230 \pm 0.017$ & $458.28 \pm 18.58$ \\
\hline DU-PW/HTPB & $0.0243 \pm 0.0007$ & $0.172 \pm 0.001$ & $75.73 \pm 0.58$ & $0.018 \pm 0.003$ & $0.240 \pm 0.017$ & $464.65 \pm 25.00$ \\
\hline
\end{tabular}

DU-PW, the weight loss of DU-PW/PW/HTPB composites gradually decreased. The weight loss of samples reduced from $0.32 \mathrm{wt} \%$ for PW/HTPB to $0.21 \mathrm{wt} \%$ for DU-PW/HTPB composites. This indicated that the 3D structure of DU can shape-stabilize the PW firmly and prevent the PW molecules from moving out of the HTPB matrix as described in Fig. 1 and verified by Fig. 4. Thus, it was revealed that DU-PW can serve as a type of shape-stabilized PW in the HTPB matrix.

\subsection{Mechanical properties of DU-PW/PW/HTPB composites}

The results of compression and tensile tests for pristine HTPB, PW/HTPB, DU-PW/PW/HTPB and DU-PW/HTPB composites are presented in Table 2. As shown in Table 2, because PW always exhibited poor mechanical properties, ${ }^{17}$ the compressive modulus, compressive strain, tensile strength and elongation at break of PW/HTPB decreased compared with the HTPB matrix. With the increasing addition of DU-PW, the compressive modulus and compressive strength of DU-PW/PW/HTPB composites increased compared with PW/HTPB. The compressive modulus and compressive strength of DU-PW/HTPB were improved as much as $1.1 \times$ and $1.8 \times$ than those of PW/HTPB. Moreover, the tensile modulus, tensile strength and elongation at break of DU-PW/PW/HTPB composites were almost consistent, while higher than those of PW/HTPB. This indicated that DU-PW can provide mechanical reinforcement for the PW/ HTPB composite, which may be attributed to the fact that the 3D structure of DU molecules can stabilize PW and firmly entangle with the HTPB matrix.

\section{Conclusions}

In this study, a novel PW-based HTPB with DU-PW PCM was proposed for prevention of PW leakage. The chemical composition, morphology, phase change performance, leakage, thermal and mechanical properties of DU-PW and DU-PW/PW/ HTPB composites were evaluated. From the results obtained, the main conclusion can be drawn is that the $T_{\mathrm{m}}$ and $T_{\mathrm{c}}$ of DUPW were enhanced by 2.84 and $1.2{ }^{\circ} \mathrm{C}$, respectively, compared with that of PW. DU-PW can be homogeneously dispersed in the HTPB matrix and effectively prevent the exudation of PW. With the addition of DU-PW, the composites can retain relatively high latent heat and good thermal reliability. Moreover, the thermal stability of DU-PW/HTPB was significantly improved compared with PW/HTPB. In addition, DU-PW can result in an enhancement of the compression properties of $\mathrm{PW} /$ HTPB. This indicated that the addition of DU-PW can improve the interaction between PW and the HTPB matrix. Consequently, DU-PW/PW/HTPB composites have a good prospect in application of PBX systems.

\section{Conflicts of interest}

The authors declare that they have no conflict of interest.

\section{Acknowledgements}

This work was supported by the National Natural Science Foundation of China (No. 20973022, 11472048, 11502249).

\section{References}

1 M. Boiocchi, P. Milova, L. Galfetti, L. D. Landro and A. K. Golovko, Progress in Propulsion Physics, 2016, vol. 8, pp. 241-262.

2 H. Abusaidi, H. R. Ghaieni, S. M. Pourmortazavi and S. H. Motamed-Shariati, J. Therm. Anal. Calorim., 2015, 124, 935-941.

3 R. L. Boddy, P. J. Gould, A. P. Jardine and D. M. Williamson, J. Dynamic Behavior Maters, 2016, 2, 157-165.

4 D. R. Kshirsagar, S. Jain, S. N. Jawalkar, N. H. Naik, S. Pawar and M. Maurya, Propellants, Explos., Pyrotech., 2016, 41, 304311.

5 C. R. Siviour, P. R. Laity, W. G. Proud, J. E. Field, D. Porter, P. D. Church, P. Gould and W. Huntingdon-Thresher, Proc. R. Soc. A, 2008, 464, 1229-1255.

6 X. Lv, M. Zha, Z. Ma, F. Zhao, S. Xu and H. Xu, Combust. Sci. Technol., 2016, 189, 312-321.

7 A. S. Alex, V. Kumar, V. Sekkar and G. G. Bandyopadhyay, J. Energ. Mater., 2016, 35, 292-299.

8 C. Bauer, Y. Metsker, C. von Sethe, M. Mutschler, M. Bambauer, P. Lungu and M. Brandl, Propulsion and Energy Forum, 2016.

9 C. K. Bina, K. G. Kannan and K. N. Ninan, J. Therm. Anal. Calorim., 2004, 78, 753-760.

10 P. Deng, Y. Liu, P. Luo, J. Wang, Y. Liu, D. Wang and Y. He, Mater. Lett., 2017, 194, 156-159. 
11 P. Deng, S. Li, S. Huang, H. Zhang, J. Wang and Y. Liu, Mater. Lett., 2018, 214, 45-49.

12 A. Mazzetti, L. Merotto and G. Pinarello, Acta Astronaut., 2016, 126, 286-297.

13 K. P. Cardoso, L. F. A. Ferrão, E. Y. Kawachi, T. B. Araújo, R. F. Nunes and M. Y. Nagamachi, J. Propul. Power, 2016, 33, 448-455.

14 M. Calabro, M. Kobald, E. Toson, H. Ciezki, S. Schlechtriem, S. di Betta, M. Coppola, L. DeLuca, L. DeLuca, S. Frolov, L. Galfetti and O. Haidn, Progress in Propulsion Physics, 2016, vol. 8, pp. 263-282.

15 G. Leccese, D. Bianchi and F. Nasuti, Propulsion and Energy Forum, 2016.

16 D.-X. Wang, S.-S. Chen, S.-H. Jin, Q.-H. Shu, Z.-M. Jiang, F.-Q. Shang and J.-X. Li, J. Energ. Mater., 2015, 34, 26-37.

17 Y. K. Sinha, B. T. N. Sridhar and R. Kishnakumar, Arabian J. Sci. Eng., 2016, 41, 4683-4690.

18 J. Giro-Paloma, M. Martínez, L. F. Cabeza and A. I. Fernández, Renewable Sustainable Energy Rev., 2016, 53, 1059-1075.

19 A. Yataganbaba, B. Ozkahraman and I. Kurtbas, Appl. Energy, 2017, 185, 720-731.

20 W. Fu, X. Liang, H. Xie, S. Wang, X. Gao, Z. Zhang and Y. Fang, Energ. Build., 2017, 136, 26-32.

21 M. Nasiri, A. R. Bahramian and H. H. Raeisi, Iran. Polym. J., 2016, 25, 1-7.

22 Y. Tang, D. Su, X. Huang, G. Alva, L. Liu and G. Fang, Appl. Energy, 2016, 180, 116-129.

23 Z. Wang, W. Situ, X. Li, G. Zhang, Z. Huang, W. Yuan, C. Yang and C. Yang, Appl. Therm. Eng., 2017, 123, 10061012.

24 C. Alkan, E. Günther, S. Hiebler, Ö. F. Ensari and D. Kahraman, Sol. Energy, 2012, 86, 1761-1769.
25 N. Sarier and E. Onder, Thermochim. Acta, 2007, 454, 90-98. 26 K. Chen, X. Yu, C. Tian and J. Wang, Energy Convers. Manage., 2014, 77, 13-21.

27 H. Wang, F. Wang, Z. Li, Y. Tang, B. Yu and W. Yuan, Appl. Energy, 2016, 176, 221-232.

28 W. G. Alshaer, M. A. Rady, S. A. Nada, E. Palomo Del Barrio and A. Sommier, Heat Mass Transfer, 2017, 53, 569-579.

29 M. Amin, N. Putra, E. A. Kosasih, E. Prawiro, R. A. Luanto and T. M. I. Mahlia, Appl. Therm. Eng., 2017, 112, 273-280.

30 T.-C. Chang, S. Lee, Y.-K. Fuh, Y.-C. Peng and Z.-Y. Lin, Appl. Therm. Eng., 2017, 112, 1129-1136.

31 J. Han and S. Liu, RSC Adv., 2017, 7, 22170-22177.

32 Z. Huang, Z. Luo, X. Gao, X. Fang, Y. Fang and Z. Zhang, Appl. Therm. Eng., 2017, 122, 322-329.

33 Q. Zhang, Z. He, X. Fang, X. Zhang and Z. Zhang, Energy Storage Materials, 2017, 6, 36-45.

34 Q. Zhang, Z. Luo, Q. Guo and G. Wu, Energy Convers. Manage., 2017, 136, 220-228.

35 S. Sundararajan, A. B. Samui and P. S. Kulkarni, Sol. Energy, 2017, 144, 32-39.

36 A. Karaipekli, A. Biçer, A. Sarı and V. V. Tyagi, Energy Convers. Manage., 2017, 134, 373-381.

37 L. Lei and S. Hongwei, Ind. Lubr. Tribol., 2011, 63, 453-455. 38 A. Sarı, C. Alkan and C. Bilgin, Appl. Energy, 2014, 136, 217227.

39 M. You, X. Wang, X. Zhang, L. Zhang and J. Wang, J. Polym. Res., 2010, 18, 49-58.

40 D. Wu, W. Wen and S. C. a. H. Zhang, J. Mater. Chem. A, 2015, 3, 2589-2600.

41 H. Li, T. Zhao and L. Wang, Chin. J. Chem., 2017, 35(7), 10791085.

42 T. Gupta and B. Adhikari, Thermochim. Acta, 2003, 402, 169181. 